\title{
Model study for the nonequilibrium magnetic domain structure during the growth of nanostructured ultrathin films
}

\author{
R. Brinzanik*, P.J. Jensen, K.H. Bennemann \\ Institut für Theoretische Physik, Freie Universität Berlin, \\ Arnimallee 14, D-14195 Berlin, Germany
}

\begin{abstract}
The nonequilibrium magnetic domain structure of growing ultrathin ferromagnetic films with a realistic atomic structure is studied as a function of coverage and temperature. We apply a kinetic Monte Carlo method to a micromagnetic model describing the transition from superparamagnetic islands at low coverages to a closed ferromagnetic film. The magnetic relaxation and the island growth happen simultaneously. Near the percolation threshold a metastable magnetic domain structure is obtained with an average domain area ranging between the area of individual magnetic islands and the area of the large domains observed for thicker ferromagnetic films. We conclude that this micro-domain structure is controlled and stabilized by the nonuniform atomic nanostructure of the ultrathin film, causing a random interaction between magnetic islands with varying sizes and shapes. The average domain area and domain roughness are determined. A maximum of the domain area and a minimum of the domain roughness are obtained as a function of the temperature.
\end{abstract}

Key words: magnetic domains, nanostructured film, nonequilibrium states, random magnet, ultrathin film growth, kinetic Monte Carlo method

PACS: 75.70.Kw, 75.60.Ch, 75.70.Ak, 75.75.+a, 75.50.Lk, 81.15.Aa

\section{Introduction}

The investigation of the magnetic domain formation, in particular in nanostructured ultrathin films, is an active field of current research. The influence of the atomic morphology on the magnetic properties is known to be especially

\footnotetext{
* Corresponding author. Tel.: +49-30-83854784; fax.: +49-30-83856799

Email address: brinzani@physik.fu-berlin.de (R. Brinzanik).
} 
strong during the initial states of the thin film growth. A small variation of the preparation conditions may change the corresponding magnetic structure markedly. This has been shown by the recent progress of highly resolving imaging techniques, which allows for the investigation of the atomic structure as well as of the magnetic domain structure of these nanostructured systems in greater detail [1-6]. For example, a strong dependence of the domain structure on the film morphology has been observed for the $\mathrm{Co} / \mathrm{Au}(111)$ thin film system yielding a smaller average domain area for rougher Co films $[1,2]$. In addition, we emphasize that the consideration of nonequilibrium states for the investigation of the magnetic domain structure is of particular importance.

In this contribution we study the interplay of the atomic and magnetic structure theoretically. In particular we simulate the spatial and temporal magnetic domain formation during the simultaneous growth of ultrathin ferromagnetic films. To our knowledge this problem has not been investigated previously. The domain structure is calculated within a kinetic Monte Carlo (KMC) simulation applied to a micromagnetic model considering exchange coupling and uniaxial lattice anisotropy. The average domain area $\bar{S}$ and the average domain roughness $\bar{R}$ of the growing thin film are calculated as functions of coverage $\Theta$ and temperature $T$. The domain roughness is defined as the edge-to-area ratio of a domain.

In general, for low coverages the growing thin film mostly consists of isolated superparamagnetic islands with a domain area of the order of the island area. On the other hand, at higher coverages the coagulation of islands results in a closed ferromagnetic film which allows for the formation of large domains with sizes of the order of about $1 \mu \mathrm{m}$, as usually observed [1]. The crossover between these two extremal domain size ranges is still not well investigated. Also, the quantities determining this transition are not known.

For the simulation of the magnetic properties of the growing thin film we consider the following important features:

i) We take into account an irregular, nanostructured film morphology which is usually present in a realistic thin film system. This is performed with the help of a simple growth model, allowing for the consideration of a size range between the atomic and the $\mu \mathrm{m}$-scale. The main quantities determining the growth (island density and -arrangement, growth mode, etc.) are chosen in accordance with experiments. Due to the strong direct exchange coupling each island is assumed to be single-domain, its magnetization rotates thus coherently [7].

ii) In the coverage range near the percolation threshold $\Theta_{P}$ of the thin film the interplay between the (irregular) atomic morphology and the magnetic domain structure is expected to be strongest. Near $\Theta_{P}$ the domain formation is governed by 'weak links' between the magnetic islands, while the island-island 
interaction tries to reach a ferromagnetic (single-domain) state of the film. The islands of a homogeneous, periodic island ensemble merge at the same time, thus the crossover from small to large domains takes place simultaneously all over the growing film. The characteristic times to cross the energy barriers, depending on the anisotropy and the island size, are the same for all islands. For an irregular nanostructure, however, the interactions between different island pairs will be strongly nonuniform (random magnet), causing various energy barriers for the magnetic rotation of different islands, and various inherent characteristic times $[8,9]$. A pre-formation of domains with intermediate areas covering several neighbouring islands is expected. This property infers much longer magnetic relaxation times to reach the thermal equilibrium than for a periodic island array. In other words, such a random ferromagnetic system can be more easily trapped into metastable states with a frozen-in multi-domain structure, and with an average domain size in between the above mentioned extremal sizes. Here the consideration of a realistic irregular atomic structure is especially important.

iii) A finite temperature is taken into account in order to investigate the domain formation of a realistic thin film system. The temperature affects strongly the probabilities of the island magnetizations to overcome the energy barriers resulting in a long range magnetic order depending on time and temperature. In addition, also the internal (short range) magnetic order of a single island due to the finite exchange coupling is considered, leading to temperature dependend island-island interactions and lattice anisotropies.

iv) Furthermore, we emphasize that for the investigation of such an ensemble of magnetic islands the consideration of states far from thermal equilibrium is very important. The long relaxation times common to a random magnet may be much larger than typical detecting times. We expect in particular that in the coverage range around $\Theta_{\mathrm{P}}$ the domain structure with an intermediate average domain area may be observable within the measuring time or the simulation time.

v) Most importantly, the equilibrium state also changes permanently, if during the magnetic relaxation the thin film grows steadily. Thus, within our simulation of the magnetic domain stucture we take simultaneously into account the temporal variation of the atomic structure during the thin film growth. This results consequently in a temporal variation of the magnetic interactions determined by the growth velocity.

The formation of large magnetic domains is expected to be facilitated by an increasing temperature, since then energy barriers can be more easily surmounted. This will result in a larger average domain area $\bar{S}$ after a given observation time, or a faster formation of larger domains. On the other hand, fluctuations at elevated temperatures will destroy large domains yielding a 
smaller average domain area. Thus, a maximum of $\bar{S}(T)$ as a function of temperature during domain formation is expected. With the same argument we expect a minimum for the average domain roughness $\bar{R}(T)$.

Previous simulations using similar micromagnetic models have been performed mainly for the investigation of the domain structure during the magnetization reversal of thicker ferromagnetic films with a granular structure [10]. In these studies the systems have been divided into periodic arrays of identical magnetic cells with a nonuniform distribution of the magnetic interactions between the cells. In contrast, within our model we consider an irregular atomic structure. Different anisotropies and magnetic island-island interactions are caused by the island size dispersion and different common surface areas between neighboring islands. To our knowledge such a realistic inhomogeneous atomic structure of an ultrathin film in the early states of film growth for the investigation of the spatial domain formation has not been considered previously.

In addition, theoretical investigations often consider equilibrium magnetic structures [11]. As mentioned, the relaxation times to reach the equilibrium states may be very long, so that in experiments metastable multi-domain structures are usually observed. In the present study we will simulate such domain patterns by calculating the temporal variation of the magnetic structure far from equilibrium. Since analytical approaches are not available for the study of the domain formation in a large system far from thermal equilibrium, we are forced to apply a kinetic (time resolved) Monte Carlo simulation.

This work is structured as follows. In Sec. 2 we outline our model. A simple growth model is applied which allows for the consideration of large systems. Magnetic interactions are taken into account by a micromagnetic model, the magnetic relaxation is determined within a kinetic Monte Carlo method. Results for the average domain area and domain roughness as functions of coverage and temperature are presented in Sec. 3. A conclusion is given in Sec. 4.

\section{MODEL}

Eden Growth Model: We apply a Monte Carlo (MC) method [12] to simulate the molecular beam epitaxial (MBE) growth of a magnetic ultrathin film on a nonmagnetic substrate using a simple solid-on-solid growth model. Within this so-called Eden model $[12,13]$ each atom is randomly attached to already existing islands, and stays immobile afterwards. To take into account different growth modes we assume furthermore that the adatoms are placed 
on lattice sites $i$ with varying probabilities

$$
p\left(q_{i}, z\right) \propto \exp \left(-A(z) \sqrt{q_{i}}\right)
$$

Here $z$ refers to the layer index. The square-root dependence on the local coordination numbers $q_{i}$ has been obtained to be approximately valid for metal surfaces [14]. By using layer dependent binding parameters $A(z)$ we are able to simulate with simple means different growth modes such as an island-type growth mode (three-dimensional islands) or a layer-by-layer growth mode. Also various surface faces and island densities and -arrangements can be considered in accordance with experiments, for example islands arranged in chains. This modified Eden growth model is valid for the case of a fast surface diffusion and a moderate step diffusion for which the mobility of the atoms is large enough to probe the different atomic positions of the island edges. Since the very time consuming calculation of the atomic diffusion is avoided, we are able to consider a large number of nonequivalent sites $\left(\sim 10^{6}\right)$. The influence of the temperature on the thin film morphology is not considered within our study [15].

In the present calculations we assume a $500 \times 500$ atomic and magnetic unit cell $\left(2.5 \cdot 10^{5}\right.$ sites $)$ on a fcc(001) lattice with periodic boundary conditions. We choose as an example the bilayer growth mode of the first two atomic layers as observed for the $\mathrm{Co} / \mathrm{Cu}(001)$ thin film system [16], using always the ratio of the binding parameters $A(1) / A(2)=0.989$. In accordance with experiment the island density is put equal to 0.005 per lattice site, refering to 1250 elementary islands in the unit cell. The simulation is started with a random distribution of occupied sites with minimal mutual distance $r_{\min }=10$ lattice constants which serve as seeds. Each additional atom is placed on a perimeter site with probability $p\left(q_{i}, z\right)$. We stop the growth after deposition of two magnetic layers. The full coverage represents a system with two completely filled layers.

Magnetic Structure: For a given atomic structure during the growth of the thin film the corresponding magnetic properties are determined by performing a kinetic Monte Carlo simulation [12]. The following micromagnetic model for the total energy of a system of interacting magnetic islands is assumed:

$$
E=-\frac{1}{4} \sum_{i, j} \gamma_{i j}(T, \Theta) L_{i j}(\Theta) \vec{S}_{i} \vec{S}_{j}-\sum_{i} K_{i}(T, \Theta) N_{i}(\Theta)\left(S_{i}^{z}\right)^{2}
$$

where $\vec{S}_{i}$ is a normalized vector characterizing the direction of the magnetization of the $i$-th island, $T$ is the temperature, and $\Theta$ the coverage of the film. Each magnetic island with $N_{i}$ atoms is assumed to be single-domain (StonerWohlfahrt particle [7]) with a single giant magnetic moment $\mu_{i}=N_{i} \mu_{\text {at }}, \mu_{\text {at }}$ 
the atomic magnetic moment. The island moments are subject to a uniaxial lattice anisotropy $K_{i}$ per atomic spin. Due to this anisotropy we simplify our calculations by considering only two different directions along the easy axis for each island moment $\left(S_{i}= \pm 1\right)$. This corresponds to the moments directed 'up' and 'down' for a perpendicular film magnetization, or to the two orientations of the in-plane magnetization in case of a rectangular (110) layer, for example. For an isolated island these two states are separated by an energy barrier given by its total lattice anisotropy $\Delta E_{i}=K_{i} N_{i}$ which has to be surmounted during magnetic reversal [17]. During the thin film growth two islands $i$ and $j$ may merge. If the directions of their magnetizations are different, the two islands minimize their mutual exchange coupling by the creation of a magnetic domain wall with the energy $E=\gamma_{i j} L_{i j}$. Here $\gamma_{i j}$ is the domain wall energy per bond and $L_{i j}(\Theta)$ is the common surface area of the islands in units of the lattice constant $a_{o}[18]$. For simplicity the magnetic dipole coupling is not considered here.

We emphasize that the energy expression Eq. (2), representing a system of individual magnetic islands with varying interactions $\gamma_{i j} L_{i j}$, is a good approximation for nanostructured systems. By application of this model we can describe isolated superparamagnetic islands at low coverages $\left(L_{i j}(\Theta)=0\right)$, as well as a strongly connected ferromagnetic film at high coverages, and the transition between these two extremes during the thin film growth. However, in the case of a smooth film (in the present study for $\Theta \gtrsim 1.8$ ) the maintenance of irregularly shaped magnetic islands with nonuniform couplings is an unphysical discretization of the system. Thus in such a coverage range our model is not valid.

Furthermore, the decreasing internal magnetic order of the islands with increasing temperature results in temperature dependend island interactions $\gamma_{i j}(T)$ and effective anisotropy coefficients $K_{i}(T)$. We treat the atomic magnetic moments as $S=1$ quantum spins. Within a mean field theory the relative internal island magnetization $m_{i}(T, \Theta)$ is given by the Brillouin function

$$
\begin{aligned}
m_{i}(T, \Theta)=S B_{1}\left(x_{i}\right) & =\frac{3}{2} \operatorname{coth}\left(\frac{3}{2} x_{i}\right)-\frac{1}{2} \operatorname{coth}\left(\frac{1}{2} x_{i}\right), \\
x_{i} & =\frac{\bar{z}_{i}(\Theta) J m_{i}(T, \Theta)}{k_{\mathrm{B}} T} .
\end{aligned}
$$

Here, $\bar{z}_{i}(\Theta)$ is the average coordination number of island $i$ which depends on the coverage $\Theta$ of the growing thin film, $k_{\mathrm{B}}$ is the Boltzmann constant. The ability of the anisotropy to maintain a certain direction of the magnetization decreases due to thermal agitation. Thus, a decreasing $m_{i}(T, \Theta)$ causes also a decreasing $K_{i}(T)$. Within a first order thermodynamic pertubation theory [19] the effective anisotropy $K_{i}(T)$ for $S=1$ is given by 


$$
\begin{aligned}
K_{i}(T, \Theta) & =K f_{i}(T, \Theta) \\
& =K\left[4-\frac{9}{2} \operatorname{coth}\left(\frac{3}{2} x_{i}\right) \operatorname{coth}\left(\frac{1}{2} x_{i}\right)+\frac{3}{2} \operatorname{coth}^{2}\left(\frac{1}{2} x_{i}\right)\right] .
\end{aligned}
$$

Note that $m_{i}(T) \rightarrow 1$ and $f_{i}(T) \rightarrow 1$ for $T \rightarrow 0$. Following the general derivation of the domain wall width [20], the influence of the finite temperature on the interaction between the islands is considered as

$$
\gamma_{i j}(T, \Theta)=\gamma \sqrt{m_{i}(T, \Theta) m_{j}(T, \Theta) f_{i j}(T, \Theta)},
$$

where $f_{i j}(T, \Theta)$ is the average of $f_{i}(T, \Theta)$ and $f_{j}(T, \Theta)$, see Eq. (5), of islands $i$ and $j$.

The temporal development of the magnetic arrangement of the island ensemble is determined as follows. Thermal activation and (surface) interaction energy between neighboring islands may cause reversals of the island magnetizations between the two states $S_{i}= \pm 1$. This relaxational behavior during the film growth is calculated with the help of the KMC method [12]. From Eq. (2) the energy of island $i$ as function of the angle $\phi$ between $\vec{S}_{i}$ and the easy axis is given by

$$
\epsilon_{i}(\phi)=E_{i} / K_{i} N_{i}=-2 h_{i} \cos \phi-\cos ^{2} \phi,
$$

with $h_{i}=S_{i} \sum_{j} \gamma_{i j} L_{i j} S_{j} / 4 K_{i} N_{i}$. Here we have made use of the condition $S_{j}= \pm 1$. By analysing Eq. (7) two cases have to be distinguished.

First for $\left|h_{i}\right| \leq 1$ the two states $S_{i}= \pm 1(\phi=0, \pi)$ both represent energy minima. These are separated by an energy barrier governed by the competing volume energy (lattice anisotropy) and surface energy contributions. The barriers for the transitions $\left(S_{i}=+1\right) \rightleftharpoons\left(S_{i}=-1\right)$ are given by $\Delta E_{i}=N_{i} K_{i}\left(h_{i} \pm 1\right)^{2}$. The flip rate $\Gamma_{i}$ of island $i$ to overcome $\Delta E_{i}$ is calculated by use of the Arrhenius-type ansatz [21]

$$
\Gamma_{i}=\Gamma_{o} \exp \left(\frac{-\Delta E_{i}}{k_{\mathrm{B}} T}\right),
$$

where the prefactor $\Gamma_{o}$ is treated as a constant 'attempt frequency', determining the time unit of the magnetic relaxation.

Secondly, for $\left|h_{i}\right|>1$ one of the states $S_{i}= \pm 1$ refers to an energy maximum and the other to a minimum. Then $\Delta E_{i}= \pm 4 N_{i} K_{i} h_{i}$ is the energy difference between the two directions of the island magnetization and is governed only by the surface energy. This case is treated with the usual Metropolis algorithm $[12,22]$, using the same prefactor $\Gamma_{o}$ as in Eq. (8). 
The growing thin film is characterized by a large amount of nonequivalent lattice sites, corresponding to a large number of different interaction parameters. Since little is known about these values, we use in our simulation averaged quantities for $\gamma, J$, and $K$ which are fixed as follows, using as an example the $\mathrm{Co} / \mathrm{Cu}(001)$ thin film system. The domain wall energy $\gamma$ is adjusted to give the observed Curie temperature of the ferromagnetic long range order of $T_{\mathrm{C}}=350 \mathrm{~K}$ of a $\mathrm{Co} / \mathrm{Cu}(001)$ film with two monolayers (ML) [23]. We obtain $\gamma=5.8 \mathrm{meV}$ per bond [18]. In a nanostructured film consisting of connected islands the internal ordering temperature of the islands $T_{\mathrm{C}}^{\text {int }}$ is larger than $T_{\mathrm{C}}$. Due to the lack of measurements for $T_{\mathrm{C}}^{\text {int }}$, the exchange coupling $J$ is chosen to yield $T_{\mathrm{C}}^{\text {int }}=450 \mathrm{~K}$ at a coverage of $2 \mathrm{ML}$, refering to a coordination number $z=8$. This results in $J=7.3 \mathrm{meV}$ per nearest neighbor bond. The uniaxial anisotropy constant is set equal to $K=0.2 \mathrm{meV}$ per atom, which is a typical value for ultrathin films [24]. For comparison, with these values the width of an undisturbed Bloch wall in a bulk ferromagnet is given by $w_{\mathrm{B}}=\pi \sqrt{J / 2 K} \approx$ $13 \mathrm{a}_{o}$, and the corresponding wall energy $\gamma_{\mathrm{B}}=4 \sqrt{J K / 2}=3.4 \mathrm{meV}$ per bond [20]. The 'attempt frequency' $\Gamma_{o}$ is set equal to $\Gamma_{o}=5.0 \cdot 10^{9} \mathrm{sec}^{-1}[21]$. The growth velocity of the film is adjusted to $1 \mathrm{ML} / 100 \mathrm{sec}$.

The simulation procedure is performed as follows: first the island seeds with random spin directions are distributed over the unit cell with periodic boundary conditions. Then for a given temperature the thin film grows up to a coverage of $2 \mathrm{ML}$, which is divided into 400 growth steps. After each growth step the quantities $N_{i}(\Theta), L_{i j}(\Theta), \gamma_{i j}(T, \Theta)$, and $K_{i}(T, \Theta)$ are calculated. By applying 100 Monte Carlo steps (MCS) per island moment of randomly chosen islands the magnetic structure of the island ensemble is allowed to relax towards its thermal equilibrium. Then the growth procedure is repeated.

The magnetic domains are determined by identifying connected islands with a parallel magnetization (Hoshen-Kopelman algorithm [25]). From this procedure the number of domains as well as their average area $\bar{S}$ and average roughness $\bar{R}$ are calculated. Only the first magnetic layer is used for this analysis. To indicate the influence of the atomic structure on the magnetic domain properties, we calculate also the average area of the elementary islands, as well as the average area of connected islands, consisting of coagulated elementary islands.

The domain roughness $R_{i}$ is defined as the ratio $R_{i}=\partial S_{i} / S_{i}$ of the number of atomic magnetic moments $\partial S_{i}$ at the outer edge of the domain and its total number $S_{i}$. Since for a domain with an increasing area its edge-to-area ratio is always decreasing, we consider here rather the average relative domain roughness $\bar{r}=\overline{R / R_{\circ}}$ with respect to the minimal roughness (smallest possible edge) $R_{\circ}^{i} \propto S_{i}^{-1 / 2}$ of a circularly shaped domain with the same area $S_{i}$. 


\section{Results and Discussion}

Using the Eden growth model, the KMC procedure, and the interaction parameters as described in the preceding section we present results for the magnetic domain structure of a growing ultrathin film. In particular, we have determined the average domain area and domain roughness as functions of the coverage and the temperature. The results are always averaged over 20 runs for systems with same global parameters determining the thin film growth and the magnetic properties.

Examples of the resulting atomic structure as obtained from our growth procedure are shown in Fig. 1, using the above described bilayer growth mode. For a better visualization of the film morphology a unit cell with $300 \times 300$ lattice sites is chosen here. Snapshots of three different coverages are depicted, $\Theta=0.5 \mathrm{ML}, 1.0 \mathrm{ML}$, and 1.5 ML. The coverage $\Theta=2.0 \mathrm{ML}$ corresponds to a smooth magnetic film with two closed layers (not shown). The resulting atomic structures are similar to those observed experimentally for the $\mathrm{Co} / \mathrm{Cu}(001)$ system [16].

In Fig. 2 we show snapshots of resulting magnetic domain structures for different coverages $\Theta$ and for the temperature $T=100 \mathrm{~K}$. As can be seen, for small coverages the domain area resembles the island area. For large coverages $\Theta \lesssim 2$ ML the thin film is almost closed and consequently the magnetic domains are very large. Very interestingly, for coverages near and above the percolation threshold $\Theta_{\mathrm{P}} \sim 0.9 \mathrm{ML}$ the domains assume an intermediate size, covering several neighboring islands. We claim that this micro-domain structure is controlled and stabilized by the nonuniform atomic nanostructure of the growing ultrathin film.

From the atomic and magnetic structures as depicted in Figs. 1 and 2 we analyse now the average domain area and domain roughness as functions of coverage and temperature. The average domain area $\bar{S}$ in units of lattice sites is given in Fig. 3(a,b) as functions of the coverage $\Theta$ and the temperature $T$. The average elementary island area $\bar{S}_{\text {island }}$ and the average area of connected islands, which serve as lower and upper limits of $\bar{S}$, are also shown in Fig. 3(a). For coverages well below the percolation threshold $\Theta_{\mathrm{P}}$ the average domain area

is of the order of $\bar{S}_{\text {island, }}$, see Fig. 2. In this case the ultrathin film consists of magnetically almost isolated islands, the exchange coupling between neighboring islands has no large influence. If no additional long range magnetic interactions such as the dipole coupling or the indirect exchange (RKKY-) coupling are important, the system of isolated magnetic islands refers to a superparamagnet. Due to their small size and their superparamagnetic behaviour the magnetic domains in this coverage range might be hardly visible with experimental means. 
With increasing coverage the islands start to merge and form large connected islands. Caused by the nonuniform island ensemble the island coalescence does not occur simultaneously in the system. In this coverage region the average domain area increases considerably, but is still markedly affected by the atomic nanostructure of the thin film. We find an average domain area definitely larger than the average elementary island area, but still much smaller than the large domain areas for an almost closed ferromagnetic film. Due to the irregular atomic structure of the island ensemble, the area of the interfaces between neighboring islands differ considerably, resulting in strongly different surface contributions of the energy barriers. Such a distribution of the effective interactions within the island ensemble can be attributed for by a 'random exchange energy' between neighboring island magnetizations. For such a random magnet metastable spin structures exhibit considerable temporal stabilities, characterized by large magnetic relaxation times [8,9]. Only after a long time the system may overcome the energy barriers and reach its equilibrium magnetic state. We expect that this mechanism also explains the appearance and stability of the magnetic micro-domain structure above the percolation threshold of the thin film as obtained from our calculations, since here the irregular atomic morphology causes long magnetic relaxation times.

In case of a uniform magnetic island structure, including also the case of a smooth ferromagnetic film, the corresponding relaxation times are much shorter and a micro-domain structure might not be observable. We have tested this assumption by simulating the domain structure also for a periodic array of identical magnetic islands with equal interactions between them, in contrast to an irregular island system. As expected, close to $\Theta_{\mathrm{P}}$ the domains increase fast to a large average size. Thus, we conclude that the frozen-in micro-domain structure with an intermediate average domain area is caused by the nonuniform island ensemble. The experimentally observed small domains in ultrathin films with a rough morphology $[1,2]$ are expected to be such a metastable, nonequilibrium magnetic structure.

With further increase of the coverage a smooth magnetic thin film is obtained, and the domain pattern is mainly determined by competing magnetic interactions (exchange, anisotropies). The domain area distribution is quite wide, few large domains coexist with a number of small domains. Due to the disappearance of these small domains, see Fig. 2(c,d), the average domain area becomes very large. Since the extension of these large domains reaches the size of our unit cell, we emphasize that in the coverage range of about 1.8 $2 \mathrm{ML}$ our simulation of the domain structure is influenced by finite size effects. As discussed in the previous section, our model should not be applied for a smooth film, which is present in this coverage range.

For the temperatures as considered in Fig. 3(a), for most coverages $\Theta$ a larger average domain area $\bar{S}(\Theta)$ is obtained for a smaller $T$. Investigating $\bar{S}(T)$ as 
a function of the temperature during the domain formation, see Fig. 3(b), we obtain a maximum of $\bar{S}(T)$ in particular at low $T$. The reason is that for low temperatures the domain formation is hindered by energy barriers. Since with an increasing $T$ the probability to overcome energy barriers becomes larger, an increasing average domain area is obtained. On the other hand, due to thermal agitation a large domain may disintegrate into domains with smaller sizes, resulting in a decreasing average domain area with increasing temperatures. While approaching the Curie temperature $T \rightarrow T_{\mathrm{C}}(2 \mathrm{ML})=350 \mathrm{~K}$ the average domain area drops considerably. With an increasing coverage $\Theta$ the maximum of $\bar{S}(T)$ is shifted to larger temperatures, since the increasing average magnetic energy between the islands is better suited to withstand thermal agitations.

In Fig. 4(a,b) we present results for the average domain roughness as functions of the coverage $\Theta$ and the temperature $T$. The domain roughness is characterized by the edge-to-area ratio of the domains. As described in Sec. 2, we present here the relative domain roughness $\bar{r}=\overline{R / R_{\circ}}$ with respect to the minimal roughness $R_{\circ}$ of circularly shaped domains. Values $\bar{r}<1$ result from single-domain states of the simulated films. Similar as for the average domain area $\bar{S}(T)$, an increasing temperature facilitates the surmount of energy barriers, and the domains may assume a more compact average shape, i.e. are less rough. In contrast, an even higher temperature will destroy ordered structures in particular at their surface. Consequently, a minimum of $\bar{r}$ as function of $T$ is observed, see Fig. 4(b). Evidently, the relative roughness depends also on the average domain area and the connectivity of the system, as can be seen from Fig. 4(a). An increasing temperature tends to smoothen the domains with a large area at larger coverages $\Theta$. For lower coverages, however, the opposite behavior is observed: a larger roughness for an increasing temperature. This might be explained by the fact that in this coverage range the average domain area and the connectivity between the islands are comparably small so that the disordering effect resulting from thermal fluctuations dominates the smoothening due to a facilitated surmount of energy barriers.

\section{Conclusion}

In this study we have investigated the magnetic domain structure of an ultrathin film in the early states of film growth. Here the influence of the film morphology on the domain formation is especially strong. A model has been applied which is suited in particular for nanostructured systems, as well as for the consideration of nonequilibrium states. To our knowledge, the occurrence and relative stability of the domain structure has not been considered previously in connection with a growing magnetic thin film [26]. 
The average domain area and the average relative domain roughness have been calculated as functions of the coverage and the temperature. In particular, we find an interesting metastable micro-domain structure near the percolation threshold of the thin film. The average area of these domains ranges between the area of the elementary islands and the large domains of a smooth ferromagnetic film. We conclude that this nonequilibrium micro-domain structure is controlled and stabilized by the nonuniform spatial (nano-) structure of the thin film. Such a mechanism could lead to the unusually small domains observed in rough ferromagnetic films $[1,2]$. Similar as in a random magnet a distribution of the magnetic couplings, as present in an irregular system, causes large magnetic relaxation times. For a thin film system with a periodic array of magnetic islands (positions, sizes, shapes, interactions), a microdomain structure might not be observable, since large domains will evolve fast due to a short relaxation time. The average domain area is found to increase with increasing coverage, and to exhibit a maximum as function of the temperature. Similarly, according to our calculations the average roughness shows a maximum as function of the coverage and a minimum as function of the temperature.

We have considered single-domain magnetic islands with a collinear magnetization during reversal (Stoner-Wohlfarth particles [7]). This approximation is valid if the island size is well below the domain wall width $w \propto \sqrt{J / K}$ of a bulk ferromagnet [20], yielding an upper limit for the island size within our calculations. For larger sizes a noncollinear island magnetization has to be taken into account [27]. Furthermore, as discussed in Sec. 2 the present model with nonuniform island interactions should not be applied for a smooth ferromagnetic film. Nevertheless, we expect that our approach is also suited for the investigation of thicker films with a considerable surface roughness.

Also other thin film magnetic quantities will be affected strongly by an irregular nanostructure and the corresponding large magnetic relaxation times. For instance, the remanent magnetization as measured e.g. by MOKE, and the magnetization reversal by an applied magnetic field may depend considerably on the nonuniform atomic structure [28]. Since in near future magnetic nanostructured systems with a defined lateral structure will be prepared in a more and more controlled manner [29], the corresponding magnetic properties can be calculated for these atomic structures. Vice versa, information about the atomic morphology can be obtained by analysing the magnetic properties.

Some remarks concerning the improvement of our present model study are in order. First, we will not only consider the two directions $S_{i} \pm 1$ of the magnetic islands, but will allow for a continuous rotation of the magnetization. This will result in particular in a noncollinear magnetic arrangement of the island ensemble. The formation of larger magnetic domains may be facilitated, resulting in a faster magnetic relaxation. Secondly, the magnetic dipole 
coupling will be included in our model, leading possibly to an even more complex magnetic structure. Thirdly, we will calculate explicitely the relaxational behavior of the magnetization for different coverages and temperatures of a nanostructured thin film. From an analysis of the temporal behavior the relaxation times can be extracted. Generally, also the growth velocity will affect the domain structure: for small velocities the islands have more time to align themselves and to create larger domains or a single-domain state. On the other hand, for a fast growth the islands become too fast too big to overcome the energy barriers, and the resulting domains are expected to be smaller [30]. An applied magnetic field will support the formation of a single-domain state. Also, we will calculate the susceptibility in response to a magnetic field, which will yield additional informations for the interplay between the atomic and the magnetic structure of growing films. Furthermore, the temperature dependence of the atomic growth can be included by applying appropriate growth mode parameters. In the present study we have used the same growth mode for all temperatures in order to concentrate solely on the temperature dependence of the magnetic properties. Finally, we will improve our KMC method by allowing for simultaneous flips of connected islands (cluster-spinflip algorithm $[12,31]$ ). The consideration of such correlated jumps will yield a more realistic relaxational behavior.

Acknowledgement: This work has been supported by the Deutsche Forschungsgemeinschaft, Sonderforschungsbereich 290, and by the Deutscher Akademischer Austauschdienst (PROCOPE).

\section{References}

[1] R. Allenspach, M. Stampanoni, and A. Bischof, Phys. Rev. Lett. 65, 3344 (1990).

[2] B. Voigtländer, G. Meyer, and N.M. Amer, Phys. Rev. B 44, 10354 (1991); R. Allenspach, J. Magn. Magn. Mater. 129, 160 (1994); M. Speckmann, H.P. Oepen, and H. Ibach, Phys. Rev. Lett. 75, 2035 (1995); R. Jansen, M. Speckmann, H.P. Oepen, and H. van Kempen, J. Magn. Magn. Mater. 165, 258 (1997).

[3] H.P. Oepen, M. Speckmann, Y. Millev, and J. Kirschner, Phys. Rev. B 55, 2752 (1997).

[4] E. Mentz, A. Bauer, D. Weiss, and G. Kaindl, Mat. Res. Soc. Symp. Proc. 475, 431 (1997).

[5] W. Kuch, J. Gilles, S.S. Kang, S. Imada, S. Suga, and J. Kirschner, Phys. Rev. B 62, 3824 (2000).

[6] A. Kubetzka, O. Pietzsch, M. Bode, and R. Wiesendanger, Phys. Rev. B 63, 140407(R) (2001). 
[7] E.C. Stoner and E.P. Wohlfarth, Trans. Roy. Soc. A 240, 599 (1948).

[8] D. Chowdhury and B. Biswal, in: D. Stauffer, ed., Annual Reviews of Computational Physics I (World Scientific, Singapore, 1994), p. 55.

[9] J.H. Oh and D.I. Choi, Phys. Rev. B 33, 3448 (1986).

[10] M. Mansuripur, J. Appl. Phys. 61, 1580 (1987); R.D. Kirby, J.X. Shen, R.J. Hardy, and D.J. Sellmyer, Phys. Rev. B 49, 10810 (1994); U. Nowak, IEEE Trans. Mag. 31, 4169 (1995).

[11] For example, in the case of an in-plane magnetization the energetically most favorable state is the single-domain state (besides closure domains at the film edges), whereas in the case of a perpendicular magnetization a stripe domain structure is the ground state. See, for instance, T. Garel and S. Doniach, Phys. Rev. B 26, 325 (1982); Y. Yafet and E.M. Gyorgy, Phys. Rev. B 38, 9145 (1988); P.J. Jensen and K.H. Bennemann, Phys. Rev. B 52, 16012 (1995).

[12] K. Binder, ed., Monte Carlo Methods in Statistical Physics (Springer-Verlag, Berlin, 1979); D.P. Landau and K. Binder, A Guide to Monte Carlo Simulations in Statistical Physics (Cambridge University Press, Cambridge, 2000)

[13] M. Eden, in: H.P. Yockey, ed., Symposium on Information Theory in Biology (Pergamon Press, New York, 1958), p. 359.

[14] M. Methfessel, D. Hennig, and M. Scheffler, Appl. Phys. A 55, 442 (1992).

[15] In principle, the temperature dependence of the binding parameters $A(z)$ is approximately given by $A(z) \sqrt{q_{i}} \propto E^{\mathrm{B}}\left(q_{i}, z\right) / k_{\mathrm{B}} T$, with $E^{\mathrm{B}}\left(q_{i}, z\right)$ the binding energy. The effect of the temperature on the thin film morphology can be indirectly taken into account by different parameters $A(z)$ and island densities.

[16] A.K. Schmid and J. Kirschner, Ultramicroscopy 42-44, 483 (1992); J. Fassbender, R. Allenspach, and U. Dürig, Surf. Sci. 383, L742 (1997); F. Nouvertné et al., Phys. Rev. B 60, 14382 (1999); P. Poulopoulos et al., submitted to Phys. Rev. B. (2001).

[17] We emphasize that we do not consider the island moments to be represented by Ising spins. Within our model we assume that each island moment rotates continuously from one state $S_{i}= \pm 1$ to the other by passing an anisotropy dependend energy barrier. Thus, in the present study the anisotropy affects the magnetical dynamic.

[18] The energy for an undisturbed Bloch domain wall in a bulk ferromagnet is given by the exchange interaction $J$ and the anisotropy $K, \gamma_{\mathrm{B}} \propto \sqrt{J \cdot K}$ [20]. The corresponding energy of a domain wall with a finite extension in a nanostructured system is in general not known. Thus we take $\gamma$ in the present work as a parameter. A recent study shows that for a constricted domain wall with atomic dimensions the wall width $w$ and the wall energy $\gamma / J$ are independent of the material parameters and is determined only by the geometry of the constriction, see P. Bruno, Phys. Rev. Lett. 83, 2425 (1999). We put $\gamma>\gamma_{\mathrm{B}}$ as calculated therein. 
[19] Y. Millev and M. Fähnle, Phys. Rev. B 51, 2937 (1995).

[20] A. Hubert and R. Schäfer, Magnetic Domains (Springer-Verlag, Berlin, 1998).

[21] L. Néel, Ann. Geophys. 5, 99 (1949); W.F. Brown, Jr., Phys. Rev. 130, 1677 (1963).

[22] N. Metropolis, A.W. Rosenbluth, M.N. Rosenbluth, A.M. Teller, and E. Teller, J. Chem. Phys. 21, 1087 (1953).

[23] U. Bovensiepen, P. Poulopoulos, W. Platow, M. Farle, and K. Baberschke, J. Magn. Magn. Mater. 192, L386 (1999).

[24] J.A.C. Bland and B. Heinrich, eds., Ultrathin Magnetic Structures I $+I I$ (Springer-Verlag, Berlin, 1994); M. Farle, B. Mirwald-Schulz, A.N. Anisimov, W. Platow, and K. Baberschke, Phys. Rev. B 55, 3708 (1997), and references therein.

[25] J. Hoshen and R. Kopelman, Phys. Rev. B 14, 3438 (1976).

[26] A chaotic domain pattern has recently been investigated theoretically for an inhomogeneous system characterized by varying magnetic interactions. For these calculations a continuum model has been applied along one spatial dimension. See N. Garcia, V.V. Osipov, E.V. Ponizovskaya, and A. del Moral, Phys. Rev. Lett. 86, 4926 (2001).

[27] D. Hinzke and U. Nowak, Phys. Rev. B 58, 265 (1998).

[28] J. Shen, M. Klaua, P. Ohresser, H. Jenniches, J. Barthel, Ch.V. Mohan, and J. Kirschner, Phys. Rev. B 56, 11134 (1997).

[29] M. Giersig and M. Hilgersdorff, J. Phys. A 32, L111 (1999); S. Sun, C.B. Murray, D. Weller, L. Folks, and A. Moser, Science 287, 1989 (2000).

[30] R. Brinzanik, P.J. Jensen, and K.H. Bennemann, Nanostruct. Mater. 12, 9 (1999).

[31] J.S. Wang and R.H. Swendsen, Physica A 167, 565 (1990); U. Wolff, Phys. Rev. Lett. 62, 361 (1989); S.W. Davis, W. McCausland, H.C. McGahagan, C.T. Tanaka, and M. Widom, Phys. Rev. E 59, 2424 (1999). 
Fig. 1. Simulation of the atomic structure of a thin film with a bilayer growth mode. The unit cell has $300 \times 300$ lattice constants and contains 450 islands (island density 0.005 islands per site). Snapshots of three different coverages are depicted, (a) $\Theta=0.5 \mathrm{ML}$, (b) $\Theta=1.0 \mathrm{ML}$, and (c) $\Theta=1.5 \mathrm{ML}$. Black refers to the uncovered substrate, light gray to the first and dark gray to the second magnetic layer. 
Fig. 2. Snapshots of magnetic domain structures at $T=100 \mathrm{~K}$ for different coverages (a) $\Theta=0.5 \mathrm{ML}$, (b) $\Theta=1.0 \mathrm{ML}$, (c) $\Theta=1.5 \mathrm{ML}$, and (d) $\Theta=2.0 \mathrm{ML}$. The unit cell has $500 \times 500$ lattice constants and contains 1250 islands (island density 0.005 islands per site). Only the domain pattern of the first magnetic layer is shown. The two gray scales refer to the two magnetic directions, the uncovered substrate is black. 
Fig. 3. Semi-logarithmic plots of the average domain area $\bar{S}$ as functions of (a) the coverage $\Theta$ and (b) the temperature $T$ during growth. In (a) the average elementary island area and the average area of connected islands are the lower and upper limits of $\bar{S}$. A nanostructured thin film system with the atomic and magnetic properties as described in the text has been assumed. The results are obtained from 20 different runs using the same growth mode and magnetic parameters. The area of the unit cell is $2.5 \cdot 10^{5}$ sites, the percolation threshold is close to $\Theta_{\mathrm{P}} \sim 0.9 \mathrm{ML}$. 
Fig. 4. Average relative domain roughness $\bar{r}=\overline{R / R_{\circ}}$ as functions of (a) the coverage $\Theta$ and (b) the temperature $T$ during growth. The domain roughness $R$ is given relative to the minimal roughness $R_{\circ}$ of a circularly shaped domain with the same area as described in Sec. 2. The results are obtained from 20 different runs using the same growth mode and magnetic parameters. Values $\bar{r}<1$ result from single domain samples. 
(a) $\Theta=0.5 \mathrm{ML}$

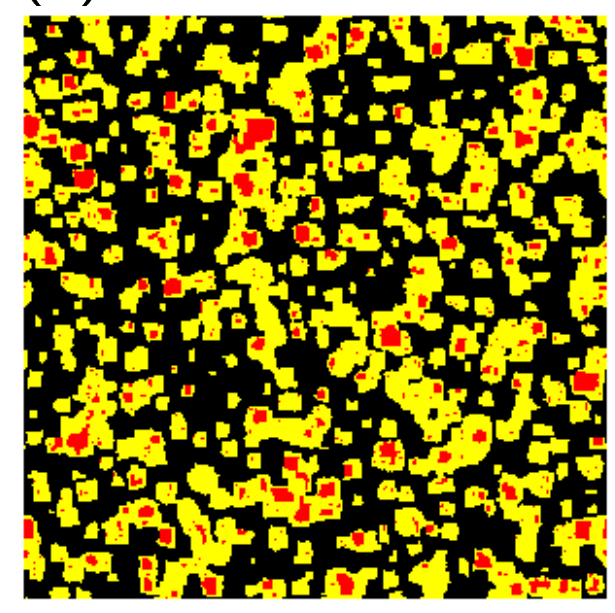

(b) $\Theta=1.0 \mathrm{ML}$

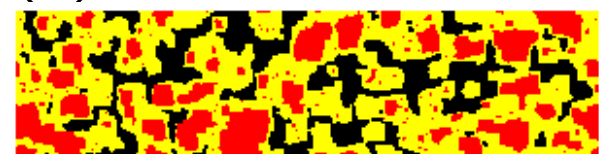

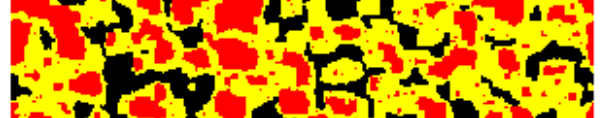

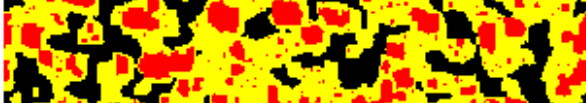

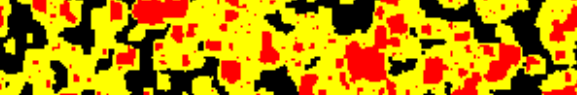

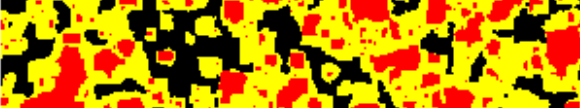

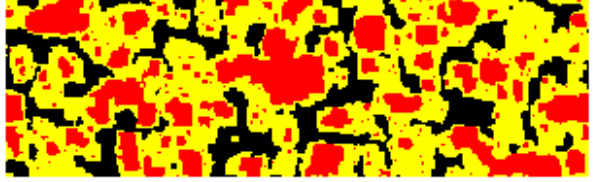

(c) $\Theta=1.5 \mathrm{ML}$

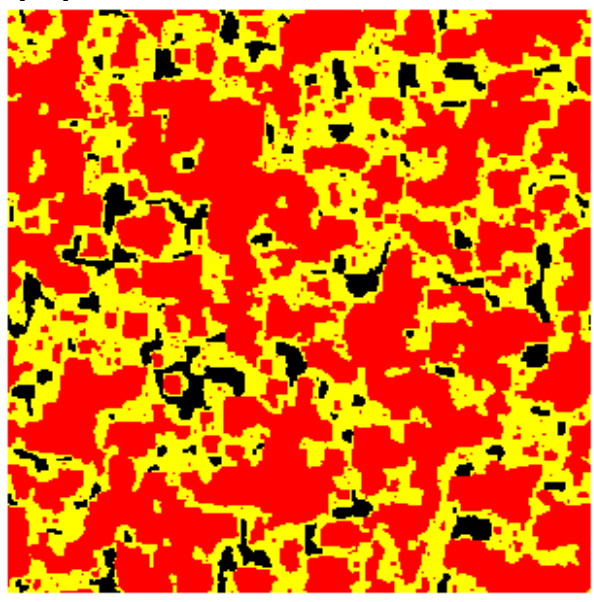


(a) $\Theta=0.5 \mathrm{ML}$

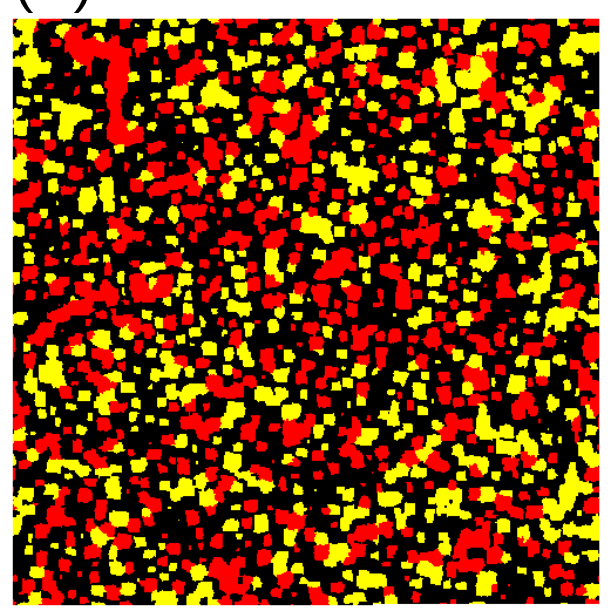

(c) $\Theta=1.5 \mathrm{ML}$

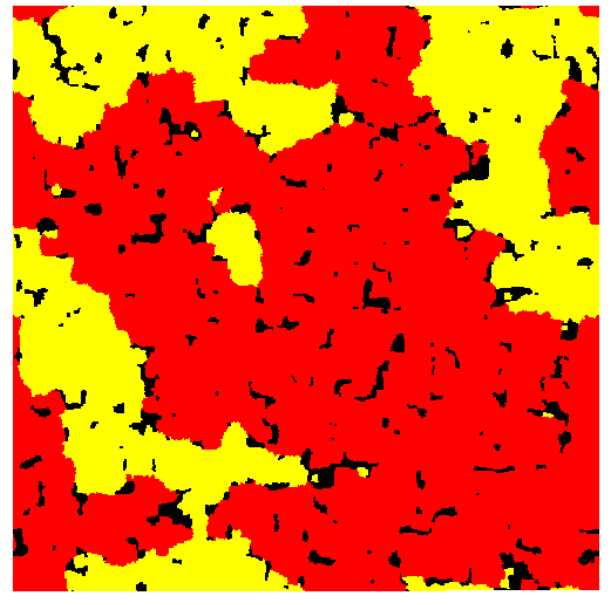

(b) $\quad \Theta=1.0 \mathrm{ML}$

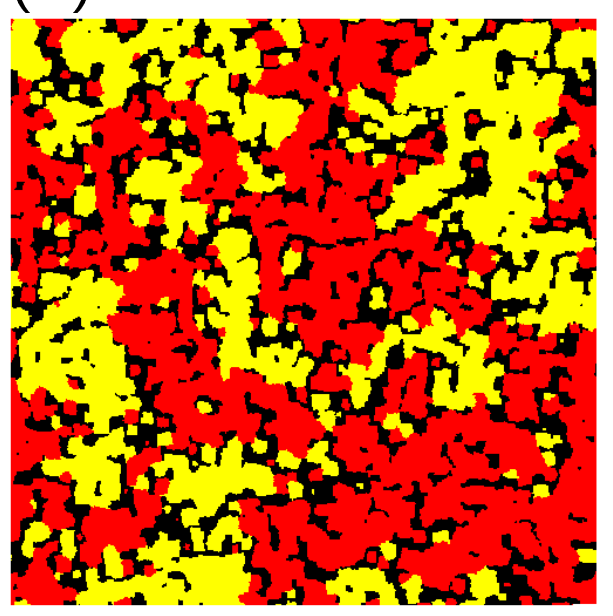

(d) $\Theta=2.0 \mathrm{ML}$

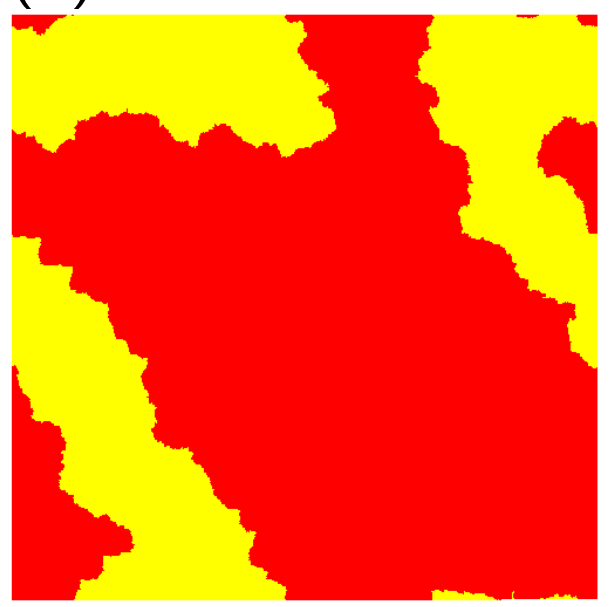



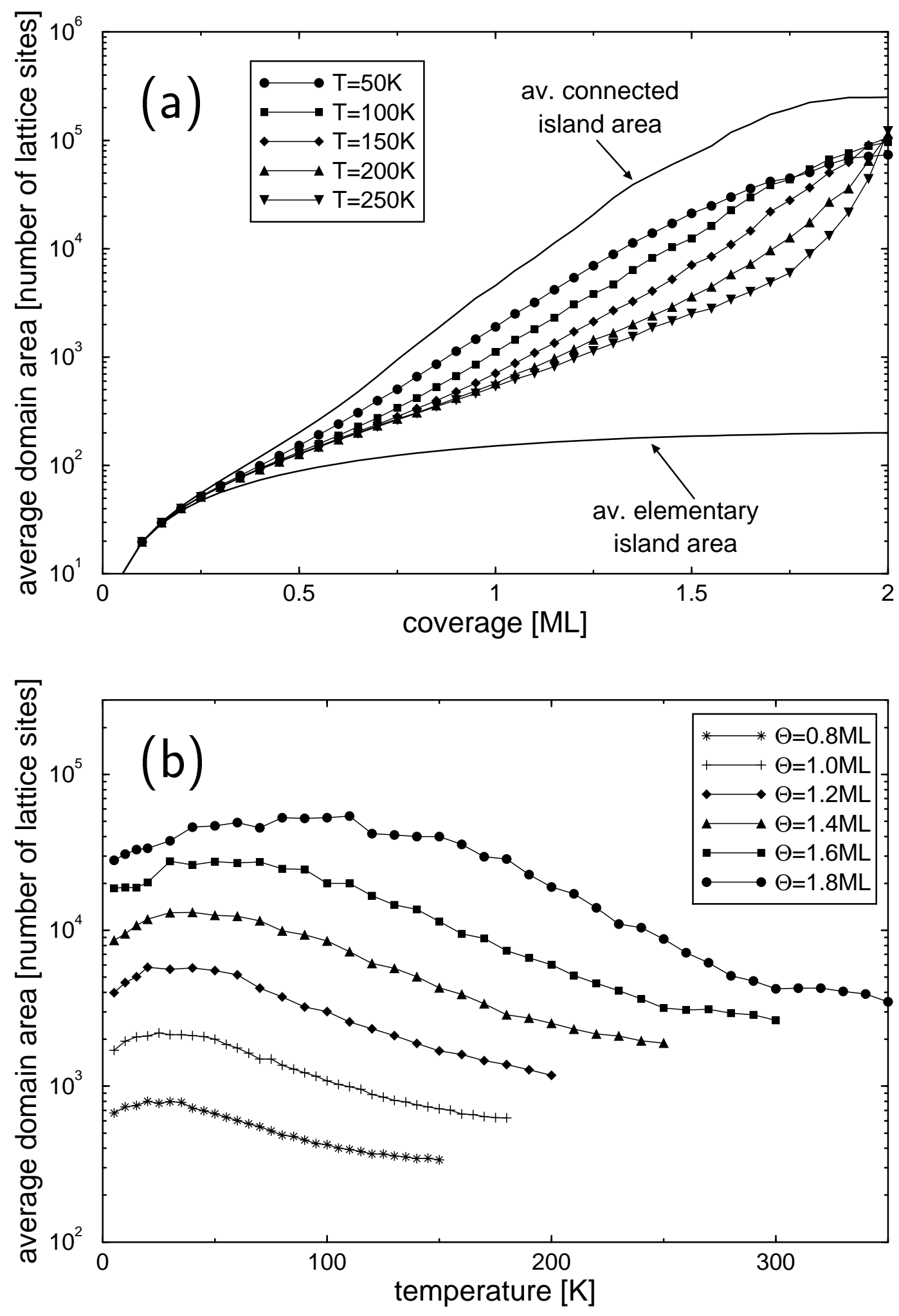

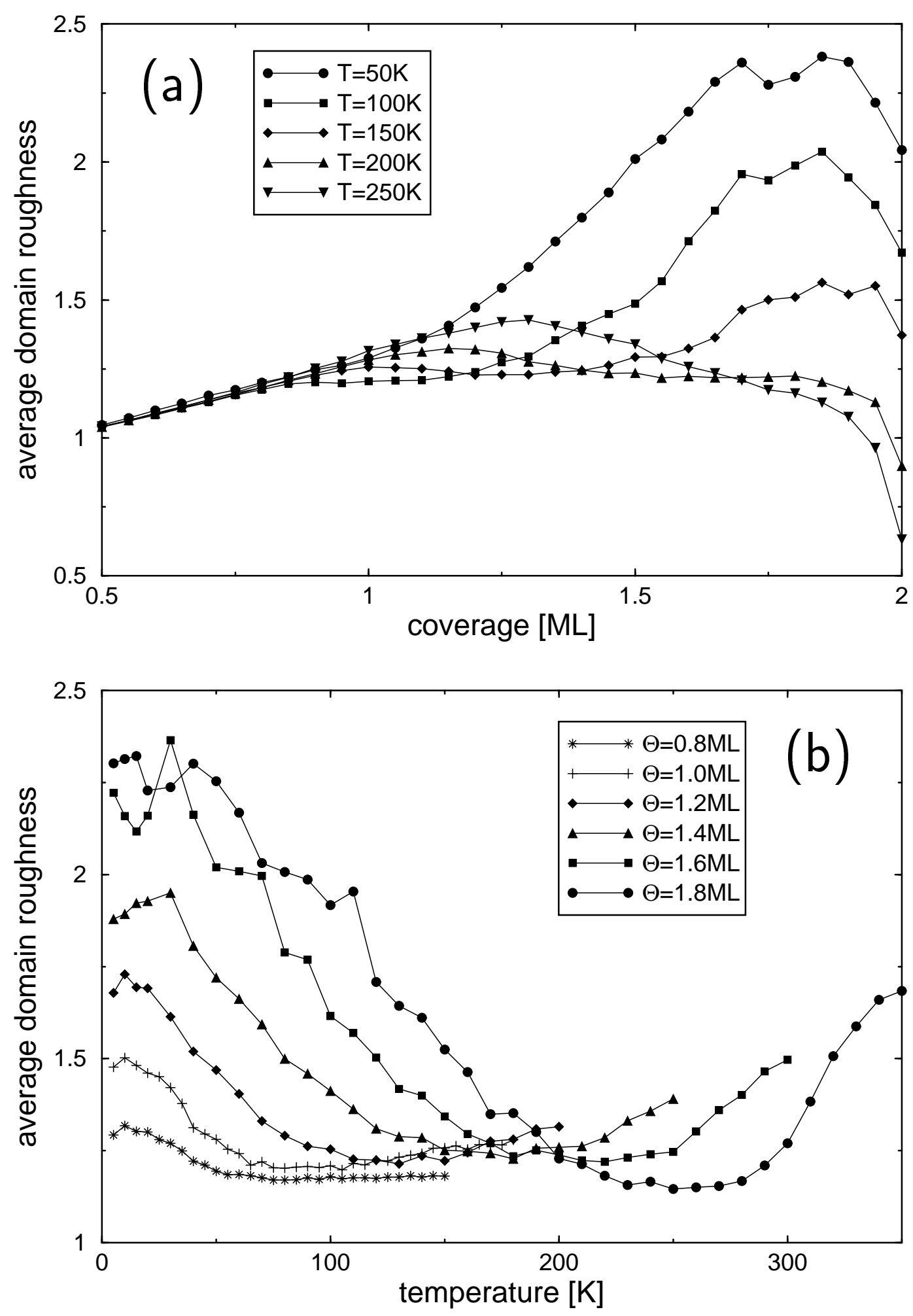